\title{
Correlations between temporal and spatial photon distributions in turbid media: Monte-Carlo modeling of time-, aperture-, angle-, and polarization-gating methods
}

\author{
Hsiang-Chieh Lee ${ }^{a}$, Cheng-Kuan Lee ${ }^{a}$, Chia-Wei Sun ${ }^{b}$, Liang Yu Yao ${ }^{a}$, Yean-Woei Kiang ${ }^{a}$ \\ and C. C. Yang ${ }^{\mathrm{a}}$ \\ ${ }^{a}$ Graduate Institute of Electro-Optical Engineering, National Taiwan University, \\ 1, Roosevelt Road, Sec. 4, Taipei, Taiwan; R.O.C. \\ (phone) 886-2-23657624 (fax) 886-2-23652637 (E-mail) ccy@cc.ee.ntu.edu.tw \\ ${ }^{b}$ Bioengineering Center, Industrial Technology Research Institute, Hsinchu, Taiwan, R.O.C.
}

\begin{abstract}
For understanding the correlations between temporal and spatial photon distributions in the process of random scattering in turbid media, the Monte Carlo algorithm is used to describe various optical gating conditions. We study the distributions of transmitted photon with time-, aperture-, and angle-gating techniques. We record the optical paths of transmitted photons with different aperture sizes and angles. Time-resolved profiles and polarization states of transmitted photons are also discussed.
\end{abstract}

\section{Introduction}

In recent years, biomedical imaging techniques have become an area of much attraction. For image quality improvement, several optical gating methods have been developed. By utilizing the dependencies of scattering characteristics on time, aperture, angle, and polarization, various imaging techniques have been explored. Among the imaging techniques of the transmission types, both snake and diffuse photons have been used for efficient imaging. Here, the snake and diffuse photons are usually defined based on different photon arrival times at a detector. Although diffuse photons are more abundant and can provide higher sensitivity in imaging, the resultant spatial resolution is usually unsatisfactory. On the contrary, the techniques based on the capture of snake photons in time gating, although suffering from low sensitivity, can provide higher resolution. Besides capturing snake photons in time gating, the methods of collecting the angle-coherent part of transmitted signals for imaging were also proposed.

In experiments, combination of more than one method was used for higher efficiency in imaging. Compared with the time-gating technique, which usually requires a short pulse laser and a time-resolved monitoring scheme, those methods are usually less expensive. For understanding more about the scattering characteristics of light passing through biological tissues, we use the Monde Carlo method for simulating various gating techniques. In particular, we are interested in the paths of photons of different gating techniques. From such studies, we can identify the photons collected for imaging among different gating methods.

\section{Monte Carlo Method}

In the Monde Carlo method, we assume light as a lot of photons. The concept of photon we use here is different from that in quantum mechanics. It simply means something carrying energy and information of polarization. The simulation is based on the concept of "photon" scattering of discrete particles of different sizes and orientations. Polarization evolution is taken care of with the calculations of Stokes vectors. We obtain each simulation result by tracing the trajectories of $10^{8}$ photons. In our model, we assume that light passes through a tissue sample of $2.5 \mathrm{~cm}$ in transmission length. In the other two directions, the sample is infinitely large. The scattering and absorption coefficients are $12.5 \mathrm{~cm}^{-1}$ and $0 \mathrm{~cm}^{-1}$, respectively. In the aperture gating method, an aperture is placed right after the sample. With different aperture radii, we can record the received powers and hence calibrate the intensity distribution at various radii. Also, by tracing the photons, which collected by the detector under a particular aperture condition, we can figure out the trajectories of photons for a certain gating condition.

\section{Simulation Results}

Fig. 1 shows the normalized optical intensity versus aperture radius with three scattering coefficients. The absorption coefficient is set zero. One can see the smooth decay of intensity with aperture radius that is quite consistent in trend with the experimental results [1]. Fig. 2 shows the time-integrated trajectories of photons of an input pulse that would be collected when a ring aperture with $0.4 \mathrm{~mm}$ in the inner radius and $0.5 \mathrm{~mm}$ in the outer radius is used. The figure shows a 2-D cross section view in our 3-D light scattering simulations. Here, one can see that the light beam spreads near the receiving end. Figs. 3(a)-(d) show the evolution of light trajectories of photons of an input pulse that would be collected when a ring aperture with $8 \mathrm{~mm}$ in the inner radius and $9 \mathrm{~mm}$ in the outer radius is used. Figs. 3(a)-(d) show the light trajectories at 9.48, 56.88, 104.28, and $151.68 \mathrm{psec}$ after the input pulse enters the sample. Here, one can see the evolution into two energy clusters, 


\section{TH3B-T2-6}

corresponding to the two apertures of a ring shape, viewed at a 2-D cross section. Other simulation results including the cases of angle and polarization gating methods will be reported in this paper during the presentation.

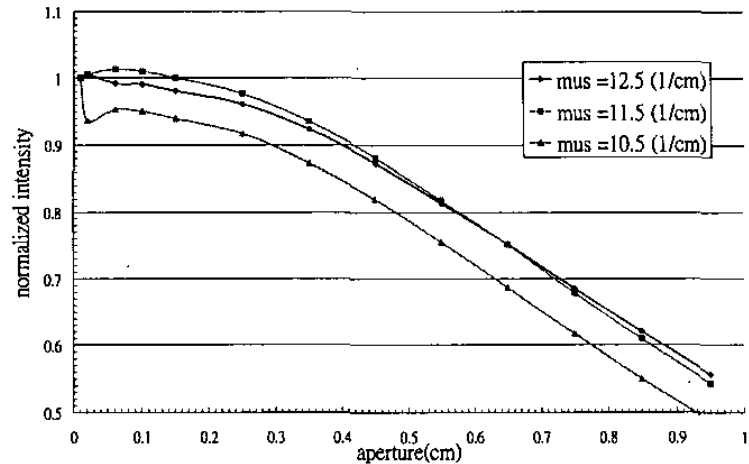

Fig. 1 Normalized intensity versus aperture radius in the aperture-gating method.

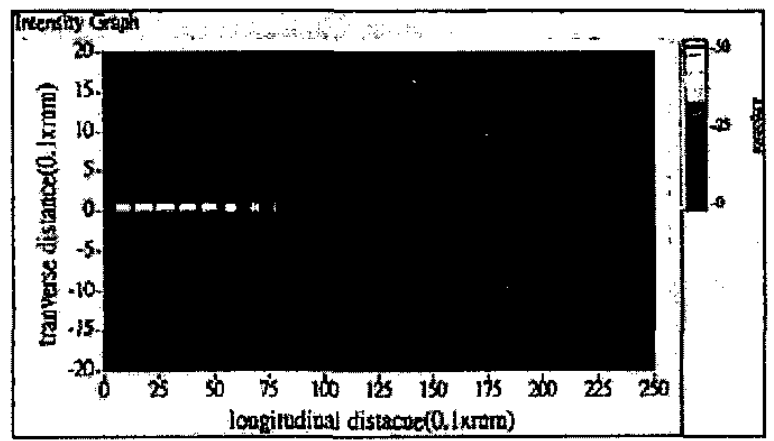

Fig. 2 Time-integrated trajectories of photons of an input pulse that would be collected when a ring aperture with $0.4 \mathrm{~mm}$ in the inner radius and $0.5 \mathrm{~mm}$ in the outer radius is used.

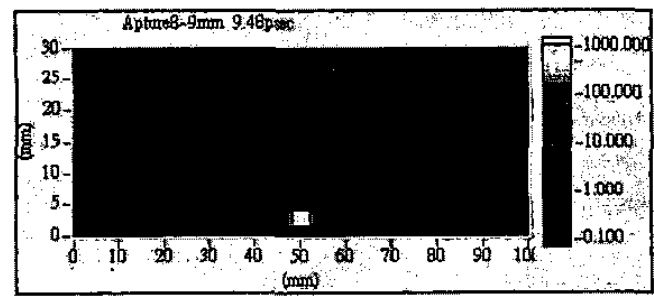

(a)

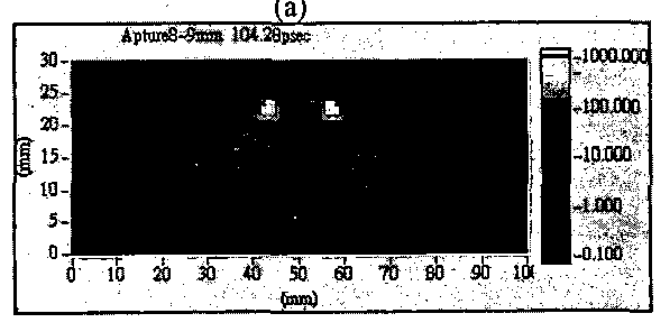

(c)

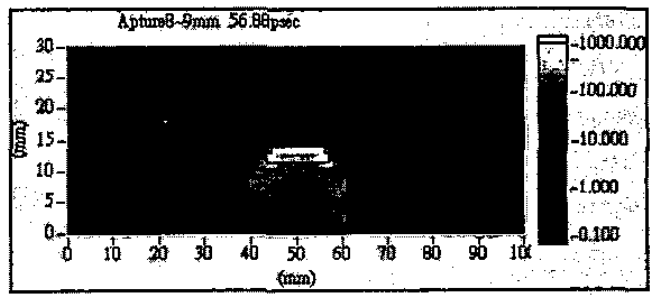

(b)

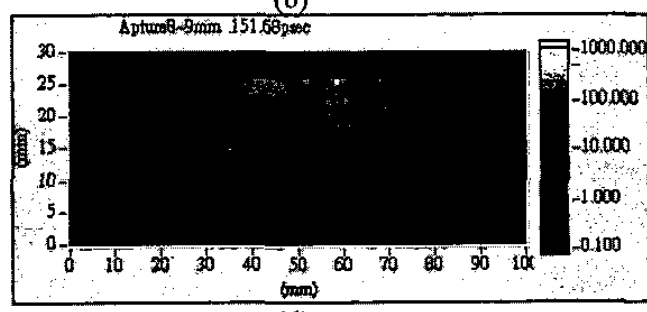

(d)

Figs. 3(a)-(d) Evolution of light trajectories of photons of an input pulse that would be collected when a ring aperture with $8 \mathrm{~mm}$ in the inner radius and $9 \mathrm{~mm}$ in the outer radius is used. Parts (a)-(d) show the light trajectories at $9.48,56.88,104.28$, and $151.68 \mathrm{psec}$ after the input pulse enters the sample.

\section{Reference}

[1] Y. M. Wang, C. W. Sun, C. K. Lee, C. W. Lu, M. T. Tsai, and C. C. Yang, Optics Express, 12, pp $1157-1168(2004)$. 